\title{
An Overview on Analyzing Deep Learning and Transfer Learning Approaches for Health Monitoring
}

\author{
Yiting Wang $\mathbb{D}^{1,2}$ Shah Nazir $\mathbb{D}^{3},{ }^{3}$ and Muhammad Shafiq ${ }^{4}$ \\ ${ }^{1}$ School of Business, Central South University, Changsha 410083, China \\ ${ }^{2}$ Hunan University of Finance and Economics, Changsha 410205, China \\ ${ }^{3}$ Department of Computer Science, University of Swabi, Khyber Pakhtunkhwa, Pakistan \\ ${ }^{4}$ Cyberspace Institute of Advance Technology, Guangzhou University, Guangzhou, China
}

Correspondence should be addressed to Yiting Wang; yitingw1@163.com and Shah Nazir; shahnazir@uoswabi.edu.pk

Received 27 January 2021; Revised 1 March 2021; Accepted 12 March 2021; Published 24 March 2021

Academic Editor: Jude Hemanth

Copyright (c) 2021 Yiting Wang et al. This is an open access article distributed under the Creative Commons Attribution License, which permits unrestricted use, distribution, and reproduction in any medium, provided the original work is properly cited.

\begin{abstract}
With the rise and advancement of technology, early detection and involvement in health-associated monitoring through home control are growing with population aging. The expansion of healthy life expectations is progressively significant due to the speedy aging of the world population. The patient requires early and home-based treatment to detect and prevent disease on time and with less effort. Home-based health monitoring has been considered the need of a smart home. The services of health monitoring can facilitate the patient by collecting and analyzing the data of health for tackling diverse complex issues of health at a large scale. Health monitoring is a sustainable progression of clinical trials for ensuring that health is monitored according to the defined protocol and standard operating procedures. Various scenarios can be considered for monitoring health and are performed through experts of the field. Healthcare systems are having large-scale infrastructure of electronic devices, medical information systems, wearable and smart devices, medical records, and handheld devices. The growth in medical infrastructure, combined with the development of computational approaches in healthcare, has empowered practitioners and researchers to devise a novel solution in the innovative spectra. A detailed report of the existing literature in terms of deep learning and transfer learning is the dire need and facilitating of modern healthcare. To overcome these limitations, therefore, the proposed study presents a comprehensive review of the existing approaches, techniques, and methods associated with deep learning and transfer learning for health monitoring. This review will help researchers to formulate new ideas for facilitating healthcare based on the existing evidence.
\end{abstract}

\section{Introduction}

The technology has ever played a significant role in healthcare and is providing facilities of being digitally transmuted. Healthcare systems are having huge infrastructure of electronic devices, medical information systems, wearable and smart devices, medical records, and handheld devices. The growth in medical infrastructure, combined with the developments of computational approaches in healthcare, has empowered practitioners and researchers to devise novel solution in innovative spectrum. The study was aimed at determining the key factors impacting the intention of individual for adopting wearable devices in healthcare [1]. Recent researches have presented a detailed overview of the existing literature for facilitating industrial Internet of Health Things [2]. A systematic literature review is reported for studying the assistance of mobile computing in healthcare [3]. The study reported the literature and presented a systematic literature review of the healthcare big data management, analytics, and its scientific programming. The report has identified 127 associated articles related to the given study for the years 2015 to 2019 [4]. The data and information of patients are warehoused in a consistent mode to certainly store, access, and retrieve the essential associated information. Smart IoT-based applications such as electronic medical report generators, wearable devices, and smart mobile phone healthcare systems have transformed the systems of conventional healthcare to the systems of digital healthcare. The 
rising advancements in healthcare has made it definitely possible for clinicians to view out and monitor the disease of patient and can provide them with easy and effective treatment including in remote areas. Mobile health (M-health) procedure has a point of view to include dire clinical administrations and came out unanimously with numerous opportunities to express extremely talented specialists.

To help minimize asset booking problems, M-health procedures require technologies, such as $5 \mathrm{G}$, Tactile, Internet, and artificial intelligence (AI). An innovative investigation of automated telehealth which depends on $5 \mathrm{G}$ Tactile Internet just as on artificial intelligence is presented. With the difficulties and problems faced in telehealth, thus, a fog-assisted intuitive model is introduced to assist and minimize the inactivity of the telehealth being experienced, thereby incorporating a fog-assisted intelligent model to assist and decrease the idleness of the cycle [5]. Detecting activities and classification through diverse sensor modalities have integrated as innovatory technologies for autonomous and real-time behavior analysis, monitoring, activities of daily living, ambient assisted living, rehabilitation, elderly care, surveillance in a smart homes, and entertainment. Smart phones, wearable devices, and ambient surroundings devices are integrated with variation of smart sensors such as magnetometer, heart rate, accelerometers, and pressure and wearable camera for detecting and monitoring activities [6]. These sensors are preprocessed, and various feature sets like frequency domain, time domain, and wavelet transform are extracted and transmuted through algorithms of machine learning for monitoring and classification of human activities. The application of deep learning to automatic feature depiction has been proposed for lessening the complexity of reliance on handcrafted features and raised performance accuracy.

The modern era of healthcare mostly depends on the use of AI such as deep learning and data science, which are the advances in the field. With the support of these at early stage detection, analysis, managements, and presentation can be made. The use of smart devices such as IoT, edge devices, drones, robots, webcam, and intelligent medical equipment is useful in the situation of pandemic [7]. Transfer learning as a domain adaptation suggests a new solution to the issue by facilitating to map feature and label structure distribution $[8,9]$.

The existing research in the field requires a detailed report of the current literature in terms of deep learning and transfer learning to facilitate modern healthcare. To overcome these limitations, therefore, the proposed study aims at presenting a wide-ranging review of the existing techniques, approaches, and methods related with deep learning and transfer learning for health monitoring. This review will benefit researchers to express novel concepts for simplifying healthcare based on the existing evidence.

The organization of the paper is given as follows: Section 2 represents the literature study of the associated researches in the area. Section 3 has discussed the fog computingbased IoT for health monitoring. Deep learning and transfer learning approaches for health monitoring approaches are briefly given in Section 4 . Section 5 has discussed the analysis of DL and TL for health monitoring. Section 6 concludes the paper.

\section{Literature Study}

Several approaches are existing for analyzing, monitoring, and managing human activities in healthcare. These approaches are providing solutions from diverse perspectives. Qureshi et al. [10] presented a systematic literature review describing the comprehensive literature on ambient supported living solutions and aids for understanding how ambient supported living motivates and helps patients with heart disease in self-management for reducing related mortality and morbidity. The study is categorized into four key themes, such as self-monitoring, wearable system, clinical management system, ambient supported living in the elderly population, and deep learning-based system to diagnose disease of the heart. Motwani et al. [11] initially presented a wide-ranging survey on ubiquitous network and systems of smart healthcare to monitor a patient with lifestyle diseases and chronic diseases. The approach presented a smart patient monitoring and recommendation as a new framework based on cloud-oriented analytics and deep learning. A case study was presented on the imbalanced dataset gathered on a patient with disorders of chronic blood pressure, and the patient status was observed. The study showed the effectiveness of the approach based on the experimental results obtained. Yao et al. [12] described the deep learning applications in healthcare. Seven areas of deep learning were focused. These areas are electrocardiography, electronic health record, community healthcare, electroencephalogram, genomics analysis and drug analysis, and data from wearable devices. Advantages and disadvantages of the existing studies are analyzed, and research challenges and future trends are discussed. Ainapure et al. [13] presented a deep learning-based crossmachine health identification approach for industrial vacuum pumps, which has gained more significance in industry and yet has not received more attention in research. The study was performed through a real-world dataset of vacuum pump, and the results shown were promising. Zhao et al. [14] presented a comprehensive review of the integrated research works of deep learning for machine health monitoring. Various perspectives were considered during the review process such as autoencoder and its variants, $\mathrm{CNN}$ and recurrent neural networks, restricted Boltzmann machine, and its variants. For performance evaluation, experimental studies were being conducted. The study has also discussed the DL-based machine health monitoring approach.

According to Hariharan and Rajkumar [15], patientgenerated health data is prepared to access a large number of information collections available on the web designed for the use of the medical service industry and 50 billion (IoT) devices to using the process of specialist and cycle-centered; the medical service industry has developed a technologyfocused combination of human innovation and medical care. This stimulates an increase of shrewd clinical equipment and extension of the total use of care for patients by drawn-out management of those clients or patients nearby. This study 
offers an analysis of the jobs of murkiness enrolling and dispersed processing; what is more, Internet of Things provides consistent setting careful organizations to the end customers and is unable to negotiate with gigantic bandwidth data on account of its inaction and volume, and in addition, moves speed when necessary current cloud computing model fog computing is designed to fix the problems that the distributed computing paradigm looks at. Debauche et al. [16] presented a cloud-based health monitoring framework for fog IoT through the use of physiological and natural signs to provide relevant information as far as daily living activities are concerned. This system empowers providers of medical care to track the state of well-being and to make adjustments to old or single persons. In addition, the approach in this paper provides a review of clinical practice and recovery times. Fog-IoT engineering consists of a remote sensor organization, a nearby input for locally and rapidly placed away information, and data handling and power lambda cloud engineering. This test provides the clinical staff the ability to view the details easily and helps them to approve the observed irregularities naturally. Finally, if a telematics split occurs, the passage continues to collect the data when performing their analysis. Clinical workers who meet up with patients sometimes send anonymized details from smart passages to the cloud for filing and for review.

\section{Fog Computing-Based IoT for Health Monitoring}

Fog computing-based IoT has facilitated healthcare to analyze, manage, and monitor human activities and has provided better solutions for caring and treatment of patients. Dhingra et al. [17] presented a study on the analysis of an incorporated mist and distributed computing structure which is acquainted with the overthrow restrictions of constant investigation, inertness, and organization blockage of essential cloud administration for traffic checking. The proposed approach is actualized to model a keen traffic observing framework. The proposed observing framework is intended for blockage checking and traffic signal administration. This can also be adjusted to detect traffic incidents that need timely assistance during obstruction. A tiny PC on module fills in as a cloud center in this scheme to collect continuous information from geologically dispersed sensors and transfer it for capacity and handling to the cloud. The results show the productivity of the mist network in improving the cloud phase presentation to reduce the reaction time and extend the capacity to move data. In addition, the planned built-in haze and cloud system is interfaced with Tweeters to deliver chaos warnings as messages to customers. The IoT and fog-based medical care system for identifying and regulating Chikungunya virus (CHV) were introduced. Fuzzy $C$-mean approaches are used to evaluate the conceivably contaminated customers and to provide customers from the mist layer with reflective and disaster concerns rapidly. From the social network analysis diagram, the flare-up job list is calculated, which speaks to any client's risk of catching or spreading the disease. It also generates an alert for government and medical service offices to monitor the CHV episode in districts that are dangerously inclined or polluted. The results of the trial feature the favorable circumstances of using both cloud measuring and distributed computing administration together to achieve the efficiency of network transmission speed, higher operational quantity, and least response time in a constant alert era compared to a single model of cloud [18]. To identify, organize, and screen customers polluted with Mosquito-Borne Diseases (MBDs), a story structure based on IoT sensors, distributed computing, and cloud computing registration is proposed. The purpose of this system is to monitor the MBD episode at an early stage. In the proposed framework, the identification factor is calculated to distinguish between MBDs, and then, the J-48 option tree classifier is used to classify each client's contamination classification. The precautionary measures are created in a split second and sent to the portable client from the mist layer if any anomaly should occur. Radio frequency recognition is used to identify the closeness of customers nearby. Fleeting network analysis uses proximity information to screen and talk to the current state of the flare-up of the MBDs. To identify the MBDs, the exploratory assessment of the proposed system resulted in high accuracy and low error rate and produced 94 percent arrangement accuracy [19].

A complete arrangement of topology control techniques for building and dealing with an enormous scope of savvy IoT city networks was proposed in this paper. A savvy IoT network is created consisting of mist doors in the construction process, and it is strengthened using assets in the system in the maintenance phase. To understand the goals, the proposed proficient calculations in each of the phases and with large reproduction based on genuine and checked IoT knowledge indexes and the adequacy of the calculations are demonstrated by contrasting and existing calculations. In the construction process, in comparison to the current calculation of the new IoT system, pick, the Hungarian-based Topology Control calculation performs better separately in terms of general framework costs and the number of necessary passages. In the maintenance process, the estimation of the vacation-based resource allocation increases the use of asset scheduling without adversely affecting the applications' inactivity requirements [20]. Another processing worldview that follows a cloud figuring strategy, named fog registering, appears to be a powerful system by expanding the cloud assets to the edge of the network to satisfy the prerequisites of constant and inactivity touchy applications in medical care. This paper considers the dedication of the paradigm of fog computing applied to medical treatment, with its key advantages in terms of inactivity, use of the network, and use of force. A fog-helping observation system is suggested in view of these boundaries, and its exhibition evaluation, moreover, is completed. The results show that this technique can be upgraded to restrict information flow in the center of the enterprise, as information is broken down locally and data protection that can be preserved locally is upgraded, the information security of patient status is strengthened, and better awareness is provided [21].

Ulcerative colitis is a genuinely normal, constant infection that causes aggravation of the considerable digestive system. This paper presents a novel IoT helping Cyber Physical 
System for conclusion and stage order of ulcerative colitis utilizing Naïve Bayes classifier and deep neural network individually. A fundamental purpose of this paper is the ongoing ready age in fog layer to the off chance that the client needs crisis treatment and on the off chance that he/she is now analyzed. The examination results and gathered clinical data of every client are put away to cloud. Usage effects of the proposed structure demonstrates its competency in the analysis and subsequent step grouping of ulcerative colitis with the haze layer continuous order portion. In addition, the warning age increases the feasibility of the system suggested [22]. Offering registration insights, stockpiling, and coordinating administration to the edge of the enterprise computing promote the benefits of distributed computing. This helps to decrease dormancy by minimizing the need for cloud delivery, continuous-discontinuous network management, enhanced security, and support of enormous machine correspondence. In this way, perception registration is a viable choice for the development of Internet of Things applications [23]. In various parts of the cloud structure, awareness methods are added, and a few special variables are considered. No detailed review of conscience methodologies occurs in mist registration, regardless of the importance of in-fog production. This paper therefore audits the momentum analysis used to ensure the figure in cloud. This paper examines the guaranteeing methods that fall into three classifications: administration/asset board, correspondence, and the executives, also application board. To choose approaches, this paper speaks to merits, bad marks, instruments, assessment types, and factors. At long last, based on the surveyed examiners, it proposes some open issues and difficulties which are worth further contemplating, furthermore, investigating in mindful methodologies in cloud figuring [24].

A business cycle model is suggested, and documentation augmentation in this article is done to enable the Internet of Things to demonstrate a conscious business step. The proposed increase considers the heterogeneous assets of IoT and non-IoT, asset limits, and existence of administration requirements. Second, it presents another cloud-based smart home engineering, which underpins the dispersed entombed and intralayer correspondence just as the constant stream handling to handle IoT data quickly also enhances the overall unwavering consistency of the system, enabling the execution of multiapplication within a cloud service architecture using the single sign-on protocol to ensure data respectability within a cloud service environment, and relies on the company and league of managers to transport BP into the required haze or potentially cloud properties. Third, a model that mentally unbalanced savvy kid and COVID-19 checking systems was introduced by using the proposed augmentation. At that point, the paper proposes the models for these two keen frameworks to do a bunch of wide research outlining the skills of this work, and, what is more, viability [25]. Karatas and Korpeoglu [26] proposed a topographically circulated multiple leveled cloud in this paper, fog registration-based IoT architecture, and proposed procedures for setting IoT information in the sections of the proposed engineering. Information is considered in various kinds, and different applications can involve each kind of

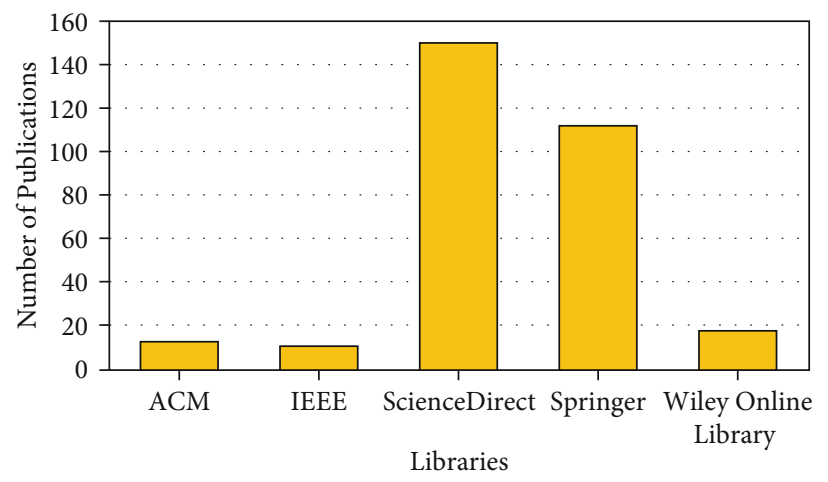

Figure 1: Overall libraries with number of articles published associated with the proposed study.

information. The model of the problem of information situation is a problem of improvement and proposed calculations for the effective, viable situation of information generated and devoured by IoT hubs that are topographically relevant. Data used for different applications is packed away in an environment that is essentially accessed by applications using that type of information for only a single period. To test the plan, comprehensive recreation trial is conducted and the results show that the design and situation techniques can productively position and store information while providing great execution to applications and organizations as far as access inertness and data transfer capability are devoted.

Bandopadhaya et al. [27] suggested a structured medical care checking response using the IoT with transmitted figures for warriors expressed in antagonistic ecological conditions. The e-care boundaries of each individual should be regularly monitored by these officers and the subsequent investigation of the dataset to be rendered with the lowest possible delay to start proper clinical assistance. In this paper, an IoT architecture arranged for three-layer administration was proposed, where the computational functionalities are appropriated between all layers. Two degrees of filtration of repetitive data having a position with secure officers have been done by the proposed circulating registering system. The filtration of the slab measure leads to a decrease in data overflowing and cloud computational weight, which increases the framework reaction time to fit crisis applications. To approve the adequacy of the proposed arrangement, a model has been developed. Bharathi et al. [28] have presented a clustering strategy focused on energy-efficient particle swarm optimization for the competitive diary well before the choice of group heads among assorted IoT gadgets. The IoT devices used to detect knowledge about medical care are assembled into a kind of bunches, and the use of energy-efficient particle swarm optimization- (PSO-) based clustering (EEPSOC) would pick a cluster head $(\mathrm{CH})$. The $\mathrm{CH}$ selected will advance the data to the cloud worker. At that point, through cloud gadgets, the $\mathrm{CH}$ is responsible for communicating IoT gadget information to the cloud worker. Near to that, a counterfeit grouping model based on neural organization is used to evaluate the cloud worker's medical care data to differentiate the severity 


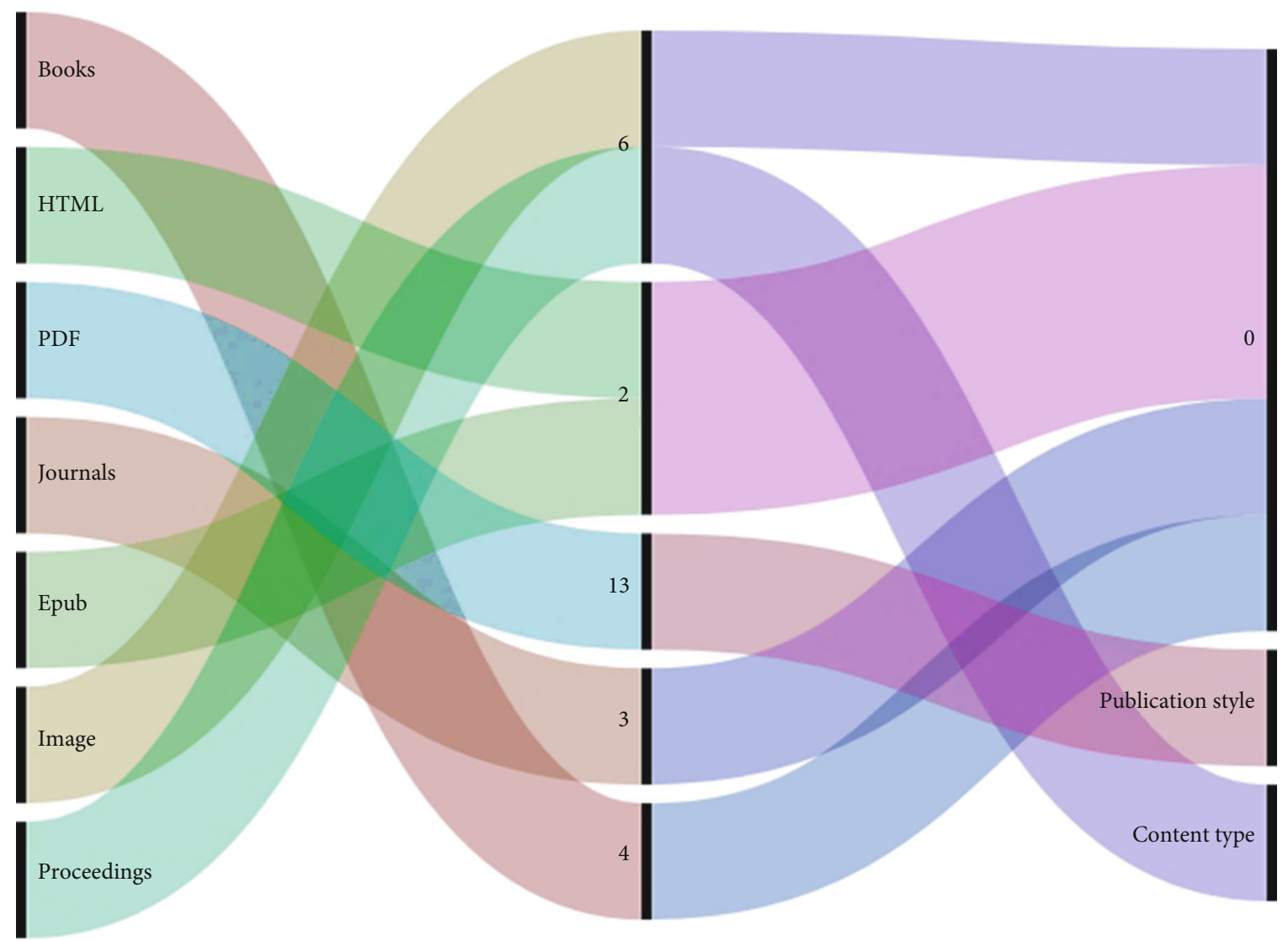

Figure 2: Publications with a number of categories.

of the disease. Using UCI data sets and restorative contraptions to anticipate the assorted understudy levels of disease intensity, an important understudy point of view medical service information is generated for experimentation. A definite relative investigation is carried out, and the reproduction outcome guaranteed, from various points of view, the credibility of the EEPSOC-ANN model over the examined techniques. Various other dimensions of healthcare are studied in research work such as the study has proposed a hybrid multicriteria decision-making approach for adopting lightweight cryptographic cipher for IoHT [29] and the evaluation of security of IoHT through the approach of AHP-TOPSIS [30].

\section{Deep Learning and Transfer Learning Approaches for Health Monitoring}

Various approaches were presented to overcome the diverse issues of healthcare monitoring. Rokni et al. [31] proposed a framework of deep learning for minimizing the expensive process of feature engineering, data labeling, and algorithm reequipping through a scalable TransNet, which is a computational method. The method learns common and reusable features in the framework lower layer and speedily reconfigures the fundamental models from a slight number of labeled instances in the new domain. The approach obtained an average accuracy of $88.1 \%$ in crosssubject learning situations through a single labeled instance for individual activity classes, and this performance enhanced to an accuracy of $92.7 \%$ with five labeled instances.

Mohammadi et al. [32] proposed the design and carrying out of noncontact sleep monitoring system analyzing the movement and posture of the body. Supervised machine learning algorithms were employed for noncontract visionbased infrared camera data through transfer learning strategy. The approach has efficiently computed the participants' poses of sleep enclosed by a blanket. The approach was assessed with manually scored sleep poses through the technique of clinical polysomnography measurement. The results of the experiments showed that the proposed approach is efficient compared to the existing approaches of videobased approaches for measuring sleep poses and generating high performance compared to the clinical standard for measuring pose through polysomnography position sensor. Shih et al. [33] offered a system which incorporates dialysis big data deep learning analysis with crossplatform physiological sensing. A timely warning dialysis discomfort including hypertension, hypotension, and cramps is precisely tackles. Brigham et al. [34] proposed an approach of neural networks to predict the parameters of future ventilation due to the high nonlinear behavior of measures of ventilation of concern and the capability of neural networks to modeling complex nonlinear functions. The experimental results of neural networks presented to be promising in its capability for predicting upcoming responses to diverse ventilation modes. Kang and Cha [35] proposed an autonomous unmanned aerial 


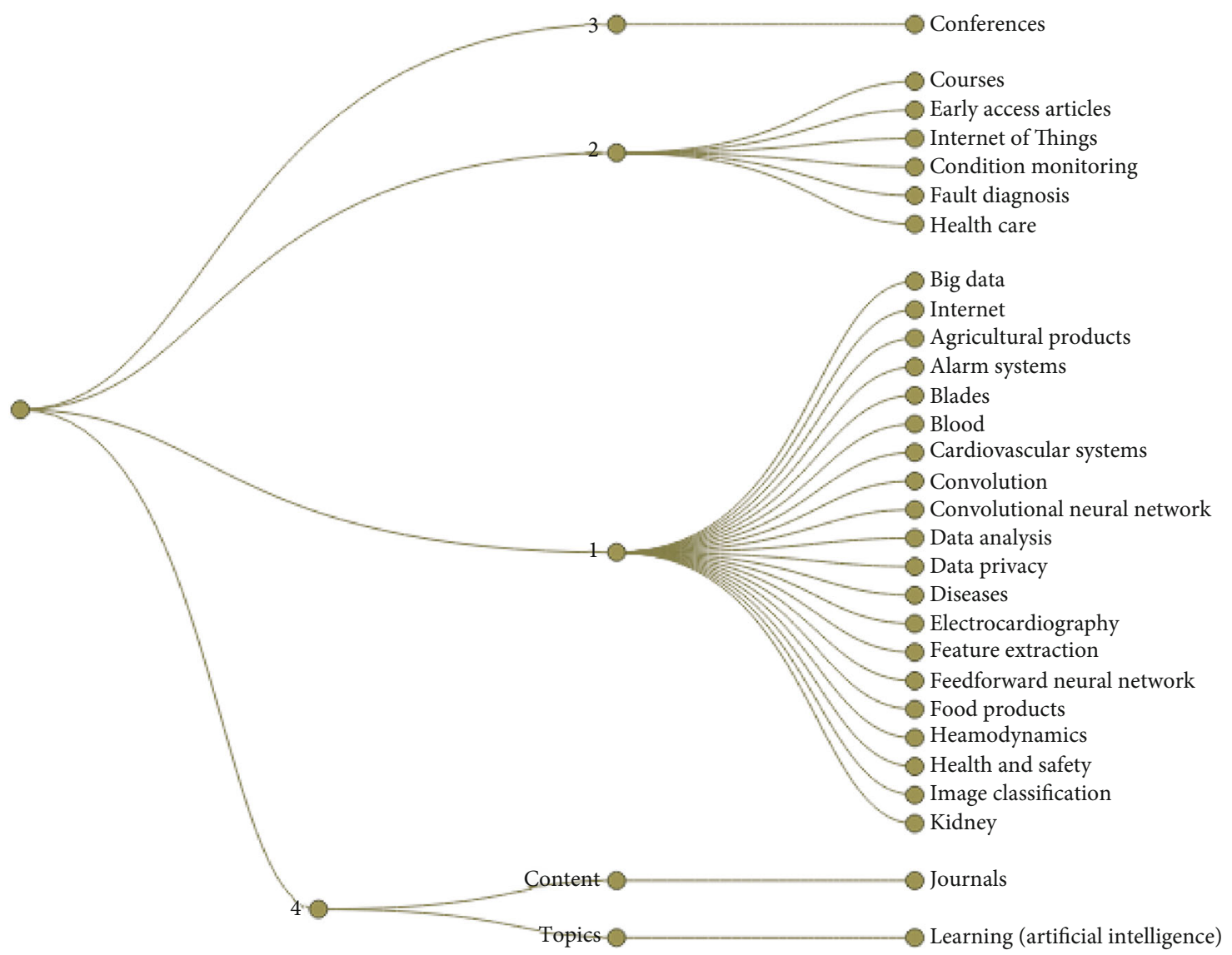

Figure 3: Content types with a total of publications.

vehicle approach through ultrasonic beacons for replacing the role of GPS; for detection of damages, a deep convolution neural network was used, and for damage localization, a geotagging approach was used. The approach has shown its effectiveness with $97.7 \%$ specificity and $91.9 \%$ sensitivity.

Azimi and Pekcan [36] proposed a convolutional neural network for monitoring of structural health exploiting a form of evaluating compressed response data through approaches of transfer learning. The employment of the proposed approach will allow the identification of damage and localization within a representative large-scale system. The approach is validated by a familiar benchmark model simulation. With the help of histograms, the CNN models are trained, and the strength of CNN approach is evaluated. Then pretrained $\mathrm{CNN}$ are fine-tuned to be adjustable with three parameters, enormously compressed response data, standard deviation based on the response mean, and a scale factor. The results showed that the transfer learning can be efficiently implemented for a system of structural health monitoring with diverse sensors. Zhu et al. [37] offered a deep learning algorithm for automatic identification of tube regions from the raw images of infrared images produced through camera data of temperature. With the help of this approach, the accurate temperature and shape of pyrolysis tubes are observed. The control limits are ultimately drawn through the $k$-NN algorithm for raising fault alarms. Kesavan and Arumugam [38] offered an approach that consists of four diverse phases such as data acquisition phase, decision-making phase, fog to cloud phase, and execution phase to transfer data to the cloud through the fog layer. Data storage and collection process are done in the first phase. The second phase consists of two diverse layers that are fog and cloud layers in which the secure incorporation of FCP is demonstrated. Feature extraction and classification process is done in the third phase. Information and notifications to the practitioner are sent in the final phase. The efficiency of the proposed approach was compared with the existing approaches, and the results provided by the proposed study were better in terms of efficiency, accuracy, computational cost, and response time. Garcia-Ceja et al. [39] designed predictive models to test user adaptive models that adapt to individual user behaviors and characteristics with condensed training data. The approaches were trained through data augmentation and deep transfer learning and were tested on two datasets; one is activity recognition dataset, and the other is emotion recombination dataset. Results of the approach have shown that it has increased significance in recognition performance with condensed data compared to the generic model.

Nweke et al. [40] presented a detailed review of the approaches of deep learning for wearable sensors and mobile-based recognition of human activities. The study has demonstrated the approach, its contribution, merits, and demerits. The study has been categorized into generative, hybrid, and discriminative approaches, and their importance 


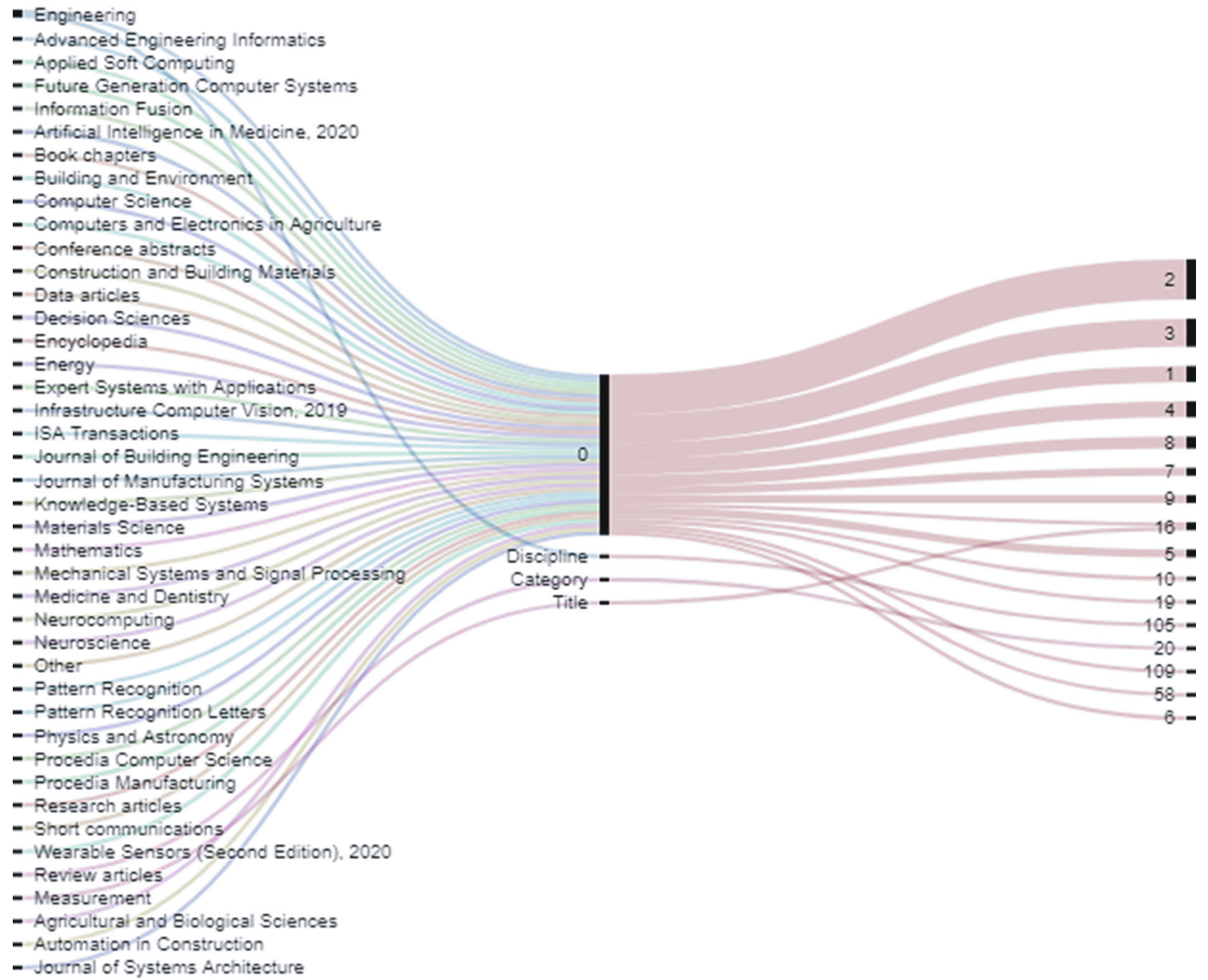

FIgURE 4: Contents with a total of publications.

is described. The study has also presented the process of evaluation and classification and demonstrated the datasets publically available for recognition of mobile sensor human activities. Some research challenges and issues were discussed. Nweke et al. [6] presented a comprehensive analysis of data fusion and various classifier system approaches for the recognition of human activities with a focus on wearable and mobile devices. The study has initially elaborated the approaches of data fusion, and modalities and feature fusion such as deep learning fusion were critically analyzed. The applications, challenges, and strengths were discussed. Various classifier systems and approaches of fusion in the literature were discussed. Sufian et al. [7] have studied the challenges and potentialities of the issues raised by pandemics. The study has demonstrated the technical background and review of the associated existing literature.

\section{Analyzing the DL and TL for Health Monitoring}

Researchers are trying to come across different approaches and mechanisms for monitoring health-associated activities. Zhang et al. [41] presented a survey of innovations in prog- nostics and health management approaches through deep learning for identification of research openings and recommended further developments. With the thorough basics of various deep learning models, the study reviewed and analyzed applications of diagnosis, fault detection, and prognosis through deep learning. The study has validated the applications of deep learning for numerous types of PHM inputs such as imagery, vibration, structured data, and time series. Various research potentials and challenges are also presented. Carlos et al. [42] offered a novel online method which is based on the tools of deep learning, rendering the idea of transfer learning for generating a framework of computational intelligence to use with the devices of IoHT. The framework can permit users for adding their images and accomplish the training of the platform easily as producing folders and employing files in the services of cloud storage. The approach has shown its robustness for people with no knowledge of programming and image processing. The approach was validated through medical databases. The experimental results showed the efficiency and reliability of the proposed approach with $91.6 \%$ accuracy in images of stroke and lung nodule databases and $92 \%$ accuracy in the skin image database. The current study has analyzed various 


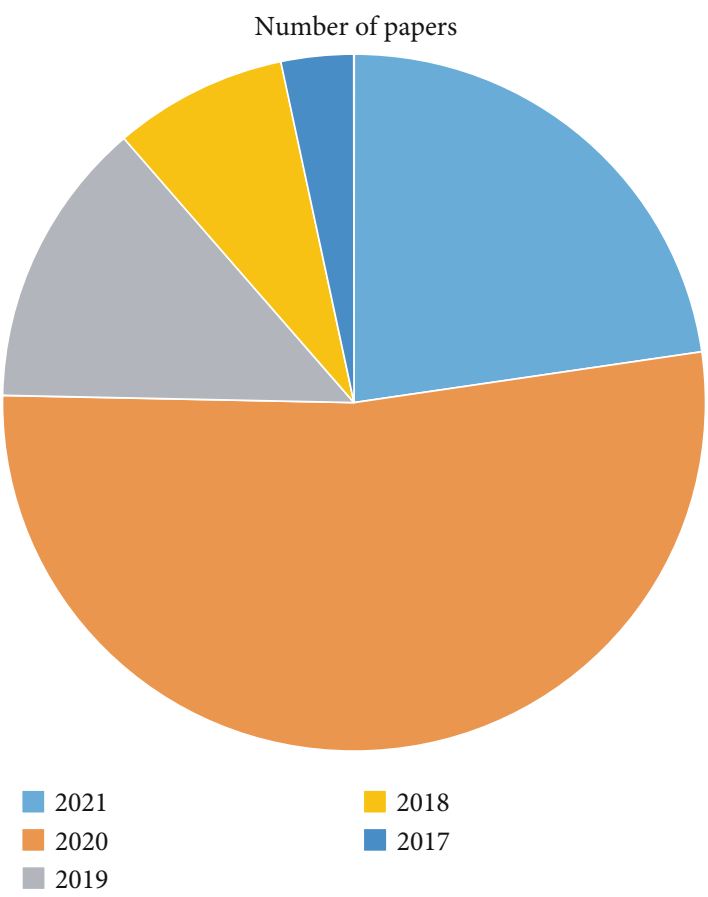

FIgURE 5: Year of publication.

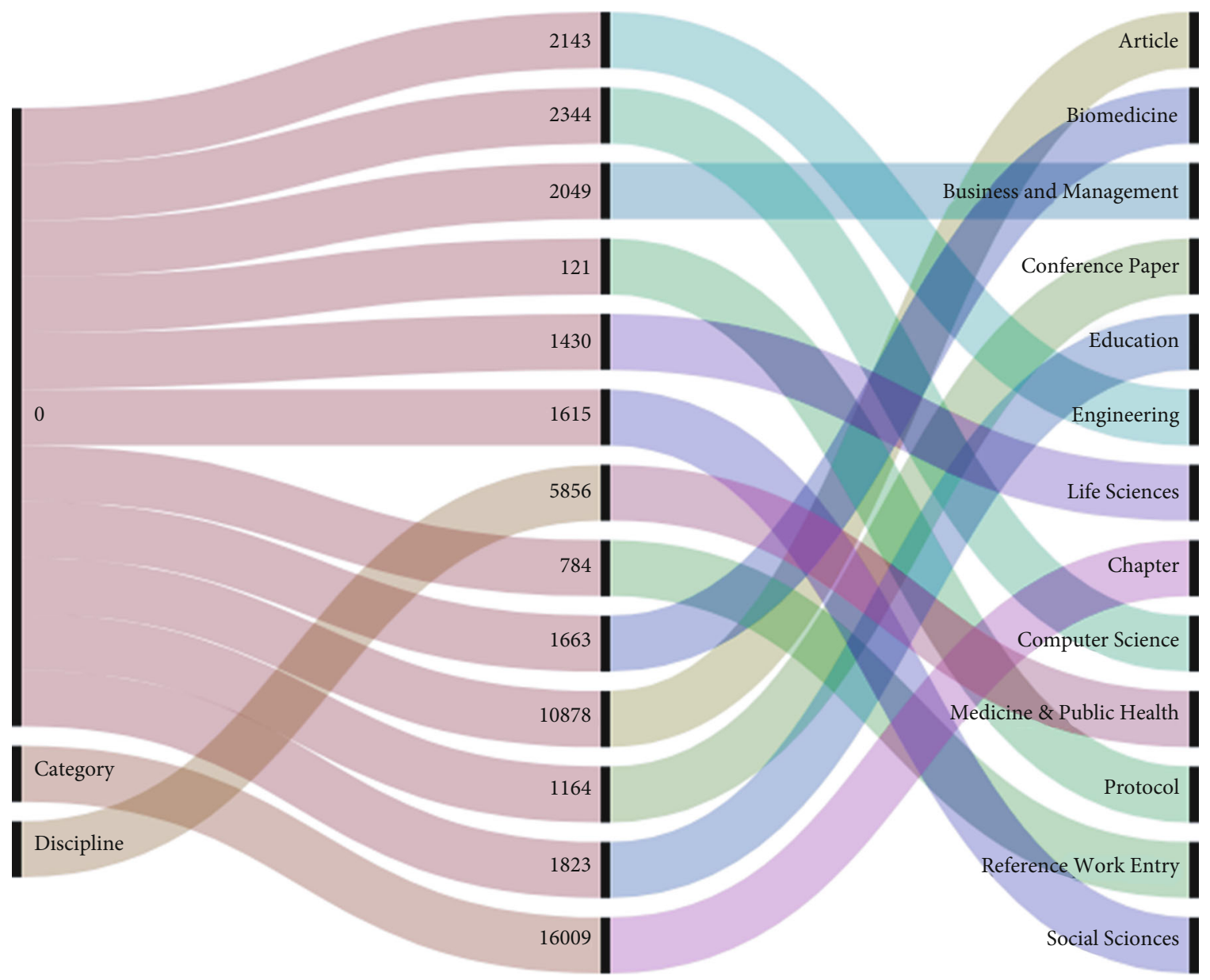

FIgUre 6: Contents with number of publications. 
libraries including ACM, IEEE, ScienceDirect, and Springer for identifying research related to deep learning and transfer learning for health monitoring. Figure 1 represents the total publications in the mentioned libraries.

After overall publication in these libraries, individual libraries were studied to identify the publications published on various types. Figure 2 depicts the contents of publications in the library of ACM.

The IEEE library was searched and the details are given in Figure 3.

The ScienceDirect library with the details are depicted in Figure 4.

The number of publications in the given years is found and is shown in Figure 5.

Figure 6 graphically shows the contents of publications in the library of Springer. The figure shows the number of publication types including article and discipline.

\section{Conclusion}

Technology has ever played a significant role in healthcare and is providing facilities for being digitally transmuted. Healthcare systems are facilitated with large-scale infrastructure of electronic and smart devices, medical information systems, wearable and smart devices, medical records, and handheld devices. The developments in medical infrastructure, combined with the progresses of computational approaches in healthcare, have allowed practitioners and researchers to develop novel solution in the state-of-the-art spectrum. The data and information of patients are warehoused in a consistent mode to certainly store, access, and retrieve the essential associated information. Smart IoTbased applications such as electronic medical report generators, wearable devices, and smart mobile phone healthcare systems have transformed the systems of conventional healthcare to the systems of digital healthcare. The rising advancements in healthcare have made it definitely possible for clinicians to view out and monitor the disease of a patient and can provide them with easy and effective treatment including in remote areas. The services of health monitoring can facilitate patient by collecting and analyzing the data of health for tackling diverse complex issue of health at a large scale. Health monitoring is a supportable development of clinical trials for ensuring that health is monitored according to the defined protocol and standard operating procedures. Various scenarios can be considered for monitoring health and are performed through expert of the field. A detail report of the existing literature in terms of deep learning and transfer learning is the dire need of modern healthcare. Therefore, the proposed study presents a comprehensive review of the existing approaches, techniques, and methods associated with deep learning and transfer learning for health monitoring. This comprehensive report will be used as evidence for devising new solutions for health monitoring.

\section{Conflicts of Interest}

The authors declare no conflict of interest.

\section{Acknowledgments}

This work was sponsored in part by the Natural Science Foundation of Hunan Province (2020JJ4121).

\section{References}

[1] S. Asadi, R. H. Abdullah, M. Safaei, and S. Nazir, "An integrated SEM-neural network approach for predicting determinants of adoption of wearable healthcare devices," Mobile Information Systems, vol. 2019, 9 pages, 2019.

[2] X. Yang, S. Nazir, H. U. Khan, M. Shafiq, and N. Mukhtar, "Parallel computing for efficient and intelligent industrial Internet of Health Things: an overview," Complexity, vol. 2021, 11 pages, 2021.

[3] S. Nazir, Y. Ali, N. Ullah, and I. García-Magariño, "Internet of Things for healthcare using effects of mobile computing: a systematic literature review," Wireless Communications and Mobile Computing, vol. 2019, Article ID 5931315, 20 pages, 2019.

[4] S. Nazir, S. Khan, H. U. K. S. Ali, I. García-Magariño, R. B. Atan, and M. Nawaz, "A comprehensive analysis of healthcare big data management, analytics and scientific programming," IEEE Access, vol. 8, pp. 95714-95733, 2020.

[5] K. Tiwari, S. Kumar, and R. K. Tiwari, "FOG assisted healthcare architecture for pre-operative support to reduce latency," Procedia Computer Science, vol. 167, pp. 1312-1324, 2020.

[6] H. F. Nweke, Y. W. Teh, G. Mujtaba, and M. A. Al-garadi, "Data fusion and multiple classifier systems for human activity detection and health monitoring: review and open research directions," Information Fusion, vol. 46, pp. 147-170, 2019.

[7] A. Sufian, A. Ghosh, A. S. Sadiq, and F. Smarandache, "A survey on deep transfer learning to edge computing for mitigating the COVID-19 pandemic," Journal of Systems Architecture, vol. 108, article 101830, 2020.

[8] P. Gardner, X. Liu, and K. Worden, "On the application of domain adaptation in structural health monitoring," Mechanical Systems and Signal Processing, vol. 138, article 106550, 2020.

[9] Y. Zhang and M. Fjeld, "Condition monitoring for confined industrial process based on infrared images by using deep neural network and variants," in Proceedings of the 2020 2nd International Conference on Image, Video and Signal Processing, Singapore, 2020.

[10] M. A. Qureshi, K. N. Qureshi, G. Jeon, and F. Piccialli, “Deep learning-based ambient assisted living for self-management of cardiovascular conditions," Neural Computing and Applications, 2021.

[11] A. Motwani, P. K. Shukla, and M. Pawar, "Novel framework based on deep learning and cloud analytics for smart patient monitoring and recommendation (SPMR)," Journal of Ambient Intelligence and Humanized Computing, 2021.

[12] Z.-J. Yao, J. Bi, and Y.-X. Chen, "Applying deep learning to individual and community health monitoring data: a survey," International Journal of Automation and Computing, vol. 15, no. 6, pp. 643-655, 2018.

[13] A. Ainapure, X. Li, J. Singh, Q. Yang, and J. Lee, "Deep learning-based cross-machine health identification method for vacuum pumps with domain adaptation," Procedia Manufacturing, vol. 48, pp. 1088-1093, 2020.

[14] R. Zhao, R. Yan, Z. Chen, K. Mao, P. Wang, and R. X. Gao, "Deep learning and its applications to machine health 
monitoring," Mechanical Systems and Signal Processing, vol. 115, pp. 213-237, 2019.

[15] U. Hariharan and K. Rajkumar, "The importance of fog computing for healthcare 4.0-based IoT solutions," in Fog Data Analytics for IoT Applications: Next Generation Process Model with State of the Art Technologies, S. Tanwar, Ed., pp. 471-494, Springer Singapore, Singapore, 2020.

[16] O. Debauche, S. Mahmoudi, P. Manneback, and A. Assila, "Fog IoT for health: a new architecture for patients and elderly monitoring," Procedia Computer Science, vol. 160, pp. 289297, 2019.

[17] S. Dhingra, R. B. Madda, R. Patan, P. Jiao, K. Barri, and A. H. Alavi, "Internet of things-based fog and cloud computing technology for smart traffic monitoring," Internet of Things, no. article 100175, 2020.

[18] S. K. Sood and I. Mahajan, "Wearable IoT sensor based healthcare system for identifying and controlling chikungunya virus," Computers in Industry, vol. 91, pp. 33-44, 2017.

[19] S. K. Sood and I. Mahajan, "Fog-cloud based cyber-physical system for distinguishing, detecting and preventing mosquito borne diseases," Future Generation Computer Systems, vol. 88, pp. 764-775, 2018.

[20] K. E. Srinivasa Desikan, V. J. Kotagi, and C. S. R. Murthy, "Topology control in fog computing enabled IoT networks for smart cities," Computer Networks, vol. 176, article 107270, 2020.

[21] P. H. Vilela, J. J. P. C. Rodrigues, P. Solic, K. Saleem, and V. Furtado, "Performance evaluation of a Fog-assisted IoT solution for e-Health applications," Future Generation Computer Systems, vol. 97, pp. 379-386, 2019.

[22] P. Verma, S. K. Sood, and H. Kaur, "A Fog-Cloud based cyber physical system for ulcerative colitis diagnosis and stage classification and management," Microprocessors and Microsystems, vol. 72, article 102929, 2020.

[23] P. Chawla and R. Chawla, "Testing perspectives of fog-based IoT applications," in Fog and Edge Computing, R. Buyya and S. N. Srirama, Eds., pp. 373-409, 2019.

[24] M. Haghi Kashani, A. M. Rahmani, and N. Jafari Navimipour, "Quality of service-aware approaches in fog computing," International Journal of Communication Systems, vol. 33, no. 8, article e4340, 2020.

[25] A. Kallel, M. Rekik, and M. Khemakhem, "IoT-fog-cloud based architecture for smart systems: prototypes of autism and COVID-19 monitoring systems," Software: Practice and Experience, vol. 51, no. 1, pp. 91-116, 2021.

[26] F. Karatas and I. Korpeoglu, "Fog-based data distribution service (F-DAD) for Internet of Things (IoT) applications," Future Generation Computer Systems, vol. 93, pp. 156-169, 2019.

[27] S. Bandopadhaya, R. Dey, and A. Suhag, "Integrated healthcare monitoring solutions for soldier using the internet of things with distributed computing," Sustainable Computing: Informatics and Systems, vol. 26, article 100378, 2020.

[28] R. Bharathi et al., "Energy efficient clustering with disease diagnosis model for IoT based sustainable healthcare systems," Sustainable Computing: Informatics and Systems, vol. 28, article 100453, 2020.

[29] L. Ning, Y. Ali, H. Ke, S. Nazir, and Z. Huanli, "A hybrid MCDM approach of selecting lightweight cryptographic cipher based on ISO and NIST lightweight cryptography secu- rity requirements for Internet of Health Things," IEEE Access, vol. 8, pp. 220165-220187, 2020.

[30] L. Wang, Y. Ali, S. Nazir, and M. Niazi, "ISA evaluation framework for security of Internet of Health Things system using AHP-TOPSIS methods," IEEE Access, vol. 8, pp. 152316152332, 2020.

[31] S. A. Rokni, M. Nourollahi, P. Alinia, I. Mirzadeh, M. Pedram, and H. Ghasemzadeh, "TransNet: minimally supervised deep transfer learning for dynamic adaptation of wearable systems," ACM Transactions on Design Automation of Electronic Systems, vol. 26, no. 1, pp. 1-31, 2021.

[32] S. M. Mohammadi, S. Enshaeifar, A. Hilton, D. J. Dijk, and K. Wells, "Transfer learning for clinical sleep pose detection using a single 2D IR camera," IEEE Transactions on Neural Systems and Rehabilitation Engineering, vol. 29, pp. 290-299, 2021.

[33] C. Shih, L. Youchen, C.-h. Chen, and W. I. C.-C. Chu, “An early warning system for hemodialysis complications utilizing transfer learning from HD IoT dataset," in 2020 IEEE 44th Annual Computers, Software, and Applications Conference (COMPSAC), pp. 759-767, Madrid, Spain, 2020.

[34] K. Brigham, S. Gupta, and J. C. Brigham, "Predicting responses to mechanical ventilation for preterm infants with acute respiratory illness using artificial neural networks," International Journal for Numerical Methods in Biomedical Engineering, vol. 34, no. 8, article e3094, 2018.

[35] D. Kang and Y.-J. Cha, "Autonomous UAVs for structural health monitoring using deep learning and an ultrasonic beacon system with geo-tagging," Computer-Aided Civil and Infrastructure Engineering, vol. 33, no. 10, pp. 885-902, 2018.

[36] M. Azimi and G. Pekcan, "Structural health monitoring using extremely compressed data through deep learning," Computer-Aided Civil and Infrastructure Engineering, vol. 35, no. 6, pp. 597-614, 2020.

[37] W. Zhu, Y. Ma, M. G. Benton, J. A. Romagnoli, and Y. Zhan, "Deep learning for pyrolysis reactor monitoring: from thermal imaging toward smart monitoring system," AIChE Journal, vol. 65, no. 2, pp. 582-591, 2019.

[38] R. Kesavan and S. Arumugam, “Adaptive deep convolutional neural network-based secure integration of fog to cloud supported Internet of Things for health monitoring system," Transactions on Emerging Telecommunications Technologies, vol. 31, no. 10, article e4104, 2020.

[39] E. Garcia-Ceja, M. Riegler, A. K. Kvernberg, and J. Torresen, "User-adaptive models for activity and emotion recognition using deep transfer learning and data augmentation," User Modeling and User-Adapted Interaction, vol. 30, no. 3, pp. 365-393, 2020.

[40] H. F. Nweke, Y. W. Teh, M. A. Al-garadi, and U. R. Alo, “Deep learning algorithms for human activity recognition using mobile and wearable sensor networks: state of the art and research challenges," Expert Systems with Applications, vol. 105, pp. 233-261, 2018.

[41] L. Zhang, J. Lin, B. Liu, Z. Zhang, X. Yan, and M. Wei, “A review on deep learning applications in prognostics and health management," IEEE Access, vol. 7, pp. 162415-162438, 2019.

[42] C. M. J. M. Dourado, S. P. P. da Silva, R. V. M. da Nobrega, P. P. Reboucas Filho, K. Muhammad, and V. H. C. de Albuquerque, "An open IoHT-based deep learning framework for online medical image recognition," IEEE Journal on Selected Areas in Communications, vol. 39, no. 2, pp. 541-548, 2021. 